\title{
Purpose of using Internet and Preference of Location for Access to Internet by Agricultural Research Scholars while using Internet of Agriculture Science in MPUAT, Udaipur (Rajasthan), India
}

\author{
L.R. Choudhary ${ }^{1 *}$, B.S. Bhimawat ${ }^{1}$ and Chamandeep Kaur ${ }^{2}$ \\ ${ }^{1}$ Department of Extension Education Jodhpur Agriculture University, Rajasthan, India \\ ${ }^{2}$ Department of Home Science, Agriculture University, Kota, Rajasthan, India
}

*Corresponding author

\section{A B S T R A C T}

\section{Keywords \\ Internet, Agriculture, \\ Research articles, Conference \\ Article Info \\ Accepted: \\ 12 January 2019 \\ Available Online: \\ 10 February 2019}

The present study was conducted at Rajasthan College of Agriculture, MPUAT, Udaipur. The RCA was selected because it is the oldest agriculture college in Rajasthan state and have adequate facilities of internet surfing for students, especially for research scholar at Central Library, departments and hostels as compared to other agriculture college in Rajasthan. Considering the importance of the internet utilization the present investigation "Attitude and Utilization Pattern of Internet among the Research Scholars of Agriculture Science in MPUAT, Udaipur Rajasthan". The sample size was 117 respondents (90 male and 27 female). The present study was conducted to analysis the purpose of using internet by Agricultural research scholar like to collect information for assignments, research reference, to send research articles for publication, information to attend seminar/conference etc.

\section{Introduction}

In India, the internet services were officially made available to public from 15th August 1995 onwards through Videsh Sanchar Nigam limited (VSNL). Today there are so many service providers offering internet services in India. There are many ways by which you can communicate with people on the internet. With the inception of Internet facility in year 1995, India brought a new era of hope and opportunities, to the people by means of information technology. It is one of the most enduring discoveries of the $20^{\text {th }}$ century, which has the potential to bring all round change in every sphere of human life. With the help of Internet one can have access of various information and data, which are earlier difficult to achieve. At that time it was a matter of great discussion, whether it is beneficial or harmful. A lot of people are concerned about the security and legitimate use of information and data available through internet. The $21^{\text {st }}$ century is witnessing a 
communication revolution with information processing and retrieving which are being reliably done at incredible speeds. The most striking invention in the field of education is the integration of Information and Communication Technology (ICT) in education. One of the most significant developments in this technology is the growth of the "Internet". The word Internet is derived from the words "global" and "network". The internet can be defined as network of networks and is the world's largest and most widely used network. According to Oxford dictionary "Internet is an international computer network connecting other networks and computers from companies, universities, NGOs etc." The internet is a useful tool for all in a technologically sophisticated world (Rehman et al., 2010).

The Internet has reshaped the way we communicate, work, play and how we understand the world around us. With the rise of the Internet as a digital highway for messages and images, people now have a fast, convenient and reliable means to transmit and receive information. Internet use is spreading rapidly into daily life and has an impact in many areas including the higher education system. Internet heralded the development and implementation of new and innovative teaching strategies in higher education institutions. Educators who advocate technology integration in learning process believe that it will improve learning and prepare students to effectively participate in the $21^{\text {st }}$ century workplace. It is now widely used as a research tool for news, education, entertainment and informal web-based education.

\section{Materials and Methods}

The study was purposively conducted in Rajasthan College of Agriculture. Udaipur due to the reason that this College is the oldest Agricultural College in Rajasthan state and enjoys more and adequate facilities of internet surfing for agricultural students, especially for research scholars at central library as well as in their respective departments and Hostels as compared to other Agricultural Colleges in Rajasthan From the RCA, Udaipur, a list of all the PG and Ph.D. research scholars was prepared from the student section of the college. There was 150 PG and 110 Ph.D research scholars, i.e. a total of 260 research scholars registered in second semester during the session 2011-12. Since the whole population was not too big, hence, the whole population as such of 260 research scholars (150 PG and 110 Ph.D research scholars) was treated as the respondents for the purpose of the study. The total size of the sample taken was 117 respondents (90 male and 27 female).

\section{Results and Discussion}

\section{Purpose of internet use}

The data presented in table 1 indicated that majority of the male agricultural research scholars used internet "to collect information for class assignments" (MS 2.6) as 65.56 per cent used it mostly, 26.89 per cent used it sometimes and 5.55 per cent had never used it. While female agricultural research scholars used internet mostly "to communicate with others" (MS 2.85) as 85.19 per cent used it mostly and 14.81 Per cent used it sometimes and these statement were ranked first. The lowest use of internet by the male agricultural research scholars was "just for pass time" (MS 1.63) as only 14.44 per cent used it mostly, 34.44 per cent used it sometimes and 51.11per cent never used it, whereas the lowest use of internet of the female agricultural research scholars was "for matrimonial purpose" (MS 1.52) as only 11.11 per cent used it mostly, 29.63 used it sometimes and 59.26 never used it, and these 
statements were placed at last position. The second lowest use of internet by male agricultural research scholars was "to purchase items" as only 17.78 per cent used it mostly, 31.11 per cent used it sometimes and 51.11 per cent never used it, whereas the second lowest use of internet by female agricultural research scholars was "to collect information for class notes" as only 14.81 per cent used it mostly, 33.33 per cent used it sometimes and 51.85 per cent never used it.
The value of rank order correlation $\left(r_{s}\right)$ was found to be 0.63 for which the calculated values of ' $t$ ' (4.01) was higher than its tabulated value at 1 per cent level of significance. Hence the null hypothesis $\left(\mathrm{H}_{02.7}\right)$ was rejected and alternate hypothesis was accepted. This leads to the conclusion that there is high significant correlation between the male and female agricultural research scholars with regard to their purpose of using Internet.

Table.1 Preference of location for access to internet by agricultural research scholars

\begin{tabular}{|c|c|c|c|c|c|c|c|c|c|c|c|c|c|}
\hline \multirow{2}{*}{ S.No. } & \multirow{2}{*}{$\begin{array}{l}\text { Location for } \\
\text { internet } \\
\text { access }\end{array}$} & \multicolumn{6}{|c|}{$\begin{array}{l}\text { Male agricultural research scholars } \\
\qquad(\mathrm{n}=90)\end{array}$} & \multicolumn{6}{|c|}{$\begin{array}{l}\text { Female agricultural research scholars } \\
\qquad(n=27)\end{array}$} \\
\hline & & Mostly & $\begin{array}{l}\text { Some } \\
\text { times }\end{array}$ & Never & Total & MS & Rank & Mostly & Sometimes & Never & Total & MS & Rank \\
\hline 1 & $\begin{array}{l}\text { College } \\
\text { library }\end{array}$ & $\begin{array}{c}54 \\
(60.00)\end{array}$ & $\begin{array}{c}27 \\
(30.00)\end{array}$ & $\begin{array}{c}9 \\
(10.00)\end{array}$ & $\begin{array}{c}90 \\
(100.00)\end{array}$ & 2.50 & I & $\begin{array}{c}7 \\
(25.93)\end{array}$ & $\begin{array}{c}12 \\
(44.44)\end{array}$ & $\begin{array}{c}8 \\
(29.63)\end{array}$ & $\begin{array}{c}27 \\
(100.00)\end{array}$ & 1.96 & II \\
\hline 2 & Home & $\begin{array}{c}15 \\
(16.67)\end{array}$ & $\begin{array}{c}21 \\
(23.33)\end{array}$ & $\begin{array}{c}54 \\
(60.00)\end{array}$ & $\begin{array}{c}90 \\
(100.00)\end{array}$ & 1.57 & V & $\begin{array}{c}5 \\
(18.52)\end{array}$ & $\begin{array}{c}11 \\
(40.74)\end{array}$ & $\begin{array}{c}11 \\
(40.74)\end{array}$ & $\begin{array}{c}27 \\
(100.00)\end{array}$ & 1.78 & III \\
\hline 3 & Department & $\begin{array}{c}23 \\
(25.56)\end{array}$ & $\begin{array}{c}36 \\
(34.44)\end{array}$ & $\begin{array}{c}31 \\
(34.44)\end{array}$ & $\begin{array}{c}90 \\
(100.00)\end{array}$ & 1.91 & III & $\begin{array}{c}14 \\
(51.85)\end{array}$ & $\begin{array}{c}0 \\
(0.00)\end{array}$ & $\begin{array}{c}13 \\
(48.15)\end{array}$ & $\begin{array}{c}27 \\
(100.00)\end{array}$ & 2.04 & I \\
\hline 4 & $\begin{array}{l}\text { Private cyber } \\
\text { café }\end{array}$ & $\begin{array}{c}18 \\
(20.00)\end{array}$ & $\begin{array}{c}30 \\
(33.33)\end{array}$ & $\begin{array}{c}42 \\
(46.67)\end{array}$ & $\begin{array}{c}90 \\
(100.00) r\end{array}$ & $n^{1.73}$ & IV & $\begin{array}{c}0 \\
(0.00)\end{array}$ & $\begin{array}{c}0 \\
(0.00)\end{array}$ & $\begin{array}{c}0 \\
(0.00)\end{array}$ & $\begin{array}{c}0 \\
(0.00)\end{array}$ & 0.00 & VII \\
\hline 5 & Hostel & $\begin{array}{c}28 \\
(31.11)\end{array}$ & $\begin{array}{c}31 \\
(34.44)\end{array}$ & $\begin{array}{c}31 \\
(34.44)\end{array}$ & $\begin{array}{c}90 \\
(100.00)\end{array}$ & 1.97 & II & $\begin{array}{c}1 \\
(3.70)\end{array}$ & $\begin{array}{c}3 \\
(11.11)\end{array}$ & $\begin{array}{c}23 \\
(85.19)\end{array}$ & $\begin{array}{c}27 \\
(100.00)\end{array}$ & 1.18 & VI \\
\hline 6 & Friends & $\begin{array}{c}16 \\
(17.78)\end{array}$ & $\begin{array}{c}36 \\
(40.00)\end{array}$ & $\begin{array}{c}38 \\
(42.22)\end{array}$ & $\begin{array}{c}90 \\
(100.00)\end{array}$ & 1.24 & VI & $\begin{array}{c}3 \\
(11.11)\end{array}$ & $\begin{array}{c}8 \\
(29.63)\end{array}$ & $\begin{array}{c}16 \\
(59.26)\end{array}$ & $\begin{array}{c}27 \\
(100.00)\end{array}$ & 1.52 & IV \\
\hline 7 & Neighborhood & $\begin{array}{c}1 \\
(1.11)\end{array}$ & $\begin{array}{c}17 \\
(18.89)\end{array}$ & $\begin{array}{c}72 \\
(80.00)\end{array}$ & $\begin{array}{c}90 \\
(100.00)\end{array}$ & 1.14 & VII & $\begin{array}{c}3 \\
(11.11)\end{array}$ & $\begin{array}{c}0 \\
(0.00)\end{array}$ & $\begin{array}{c}24 \\
(88.89)\end{array}$ & $\begin{array}{c}27 \\
(100.00)\end{array}$ & 1.22 & V \\
\hline
\end{tabular}

Rank correlation coefficient $\left(\mathrm{r}_{\mathrm{s}}\right)=0.25 \quad \mathrm{t}=0.58$ (Non significant at 0.05 level of probability)

Tabulated value of $t$ at 0.05 level of probability with 5 degrees of freedom $=2.57$ 
Table.2 Purpose of using internet by agricultural research scholars

$\mathrm{n}=117$

\begin{tabular}{|c|c|c|c|c|c|c|c|c|c|c|c|}
\hline \multirow[t]{2}{*}{$\begin{array}{l}\text { S. } \\
\text { No. }\end{array}$} & \multirow[t]{2}{*}{$\begin{array}{l}\text { Purpose of using } \\
\text { internet }\end{array}$} & \multicolumn{5}{|c|}{$\begin{array}{l}\text { Male agricultural research scholars } \\
\qquad(\mathrm{n}=90)\end{array}$} & \multicolumn{5}{|c|}{$\begin{array}{l}\text { Female agricultural research scholars } \\
\qquad(n=27)\end{array}$} \\
\hline & & Mostly & $\begin{array}{l}\text { Some } \\
\text { times }\end{array}$ & Never & MS & Rank & Mostly & Sometimes & Never & MS & Rank \\
\hline 1 & $\begin{array}{l}\text { To collect information } \\
\text { for class assignments }\end{array}$ & $\begin{array}{c}59 \\
(65.56\end{array}$ & $\begin{array}{c}26 \\
(26.89)\end{array}$ & $\begin{array}{c}5 \\
(5.55)\end{array}$ & 2.6 & I & $\begin{array}{c}15 \\
(55.560\end{array}$ & $\begin{array}{c}12 \\
(44.44)\end{array}$ & $\begin{array}{c}0 \\
(0.00)\end{array}$ & 2.56 & IV \\
\hline 2 & $\begin{array}{l}\text { To collect information } \\
\text { for research reference }\end{array}$ & $\begin{array}{c}57 \\
(63.33)\end{array}$ & $\begin{array}{c}28 \\
(31.11)\end{array}$ & $\begin{array}{c}5 \\
(5.55)\end{array}$ & 2.58 & II & $\begin{array}{c}22 \\
(81.48)\end{array}$ & $\begin{array}{c}5 \\
(18.52)\end{array}$ & $\begin{array}{c}0 \\
(0.00)\end{array}$ & 2.81 & II \\
\hline 3 & $\begin{array}{l}\text { To send research article } \\
\text { for publication }\end{array}$ & $\begin{array}{c}31 \\
(34.44)\end{array}$ & $\begin{array}{c}32 \\
(35.55)\end{array}$ & $\begin{array}{c}27 \\
(30.00)\end{array}$ & 2.04 & XIX & $\begin{array}{c}7 \\
(25.93)\end{array}$ & $\begin{array}{c}8 \\
(29.63)\end{array}$ & $\begin{array}{c}12 \\
(44.44)\end{array}$ & 1.70 & XXIII \\
\hline 4 & $\begin{array}{l}\text { To collect information } \\
\text { for higher education. }\end{array}$ & $\begin{array}{c}42 \\
(46.67)\end{array}$ & $\begin{array}{c}38 \\
(42.22))\end{array}$ & $\begin{array}{c}10 \\
(11.11)\end{array}$ & 2.36 & VIII & $\begin{array}{c}5 \\
(18.52)\end{array}$ & $\begin{array}{c}19 \\
(70.37)\end{array}$ & $\begin{array}{c}3 \\
(11.11)\end{array}$ & 2.07 & XIX \\
\hline 5 & $\begin{array}{l}\text { To collect information } \\
\text { for abroad studies }\end{array}$ & $\begin{array}{c}23 \\
(25.56)\end{array}$ & $\begin{array}{c}47 \\
(52.22)\end{array}$ & $\begin{array}{c}20 \\
(22.22)\end{array}$ & 2.03 & XX & $\begin{array}{c}7 \\
(25.93)\end{array}$ & $\begin{array}{c}12 \\
(44.44)\end{array}$ & $\begin{array}{c}8 \\
(29.63)\end{array}$ & 1.96 & $\mathrm{XX}$ \\
\hline 6 & $\begin{array}{l}\text { To collect information } \\
\text { to attend } \\
\text { seminar/conference }\end{array}$ & $\begin{array}{c}36 \\
(40.00)\end{array}$ & $\begin{array}{c}41 \\
(45.56)\end{array}$ & $\begin{array}{c}13 \\
(14.44)\end{array}$ & 2.26 & XIII & $\begin{array}{c}11 \\
(40.74)\end{array}$ & $\begin{array}{c}12 \\
(44.44)\end{array}$ & $\begin{array}{c}4 \\
(14.82)\end{array}$ & 2.26 & XIII \\
\hline 7 & $\begin{array}{l}\text { Searching useful sites } \\
\text { for career development }\end{array}$ & $\left(\begin{array}{c}45 \\
(50.00\end{array}\right.$ & $\begin{array}{c}38 \\
(42.22)\end{array}$ & $\begin{array}{c}7 \\
(7.78)\end{array}$ & 2.42 & VI & $\begin{array}{c}12 \\
(44.44)\end{array}$ & $\begin{array}{c}15 \\
(55.56)\end{array}$ & $\begin{array}{c}0 \\
(0.00)\end{array}$ & 2.44 & V \\
\hline 8 & $\begin{array}{l}\text { For preparation for } \\
\text { competitive exam }\end{array}$ & $\begin{array}{c}36 \\
(40.00)\end{array}$ & $\begin{array}{c}45 \\
(50.00)\end{array}$ & $\begin{array}{c}9 \\
(10.00)\end{array}$ & 2.30 & IX & $\begin{array}{c}12 \\
44.44\end{array}$ & $\begin{array}{c}12 \\
(44.44)\end{array}$ & $\begin{array}{c}3 \\
(14.82)\end{array}$ & 2.33 & XII \\
\hline 9 & $\begin{array}{l}\text { For generating self } \\
\text { employment }\end{array}$ & $\begin{array}{c}27 \\
(30.00)\end{array}$ & $\begin{array}{c}42 \\
46.67\end{array}$ & $\begin{array}{c}21 \\
23.33\end{array}$ & 2.06 & XVIII & $\begin{array}{c}14 \\
(51.85)\end{array}$ & $\begin{array}{c}8 \\
(29.63)\end{array}$ & $\begin{array}{c}5 \\
(18.52)\end{array}$ & 2.33 & XI \\
\hline 10 & $\begin{array}{l}\text { To send application for } \\
\text { job }\end{array}$ & $\begin{array}{c}42 \\
(46.67)\end{array}$ & $\begin{array}{c}41 \\
(45.55)\end{array}$ & $\begin{array}{c}7 \\
(7.78)\end{array}$ & 2.39 & VII & $\begin{array}{c}11 \\
(40.74)\end{array}$ & $\begin{array}{c}15 \\
(15.55)\end{array}$ & $\begin{array}{c}1 \\
(3.70)\end{array}$ & 2.37 & VI \\
\hline 11 & For entertainment & $\begin{array}{c}39 \\
(43.33)\end{array}$ & $\begin{array}{c}37 \\
(15.56)\end{array}$ & $\begin{array}{c}14 \\
(15.56)\end{array}$ & 2.28 & $X$ & $\begin{array}{c}4 \\
(14.82)\end{array}$ & $\begin{array}{c}22 \\
(81.48)\end{array}$ & $\begin{array}{c}1 \\
(3.70)\end{array}$ & 2.11 & XVIII \\
\hline 12 & $\begin{array}{l}\text { To e-mail to friend \& } \\
\text { relative }\end{array}$ & $\begin{array}{c}44 \\
(48.89)\end{array}$ & $\begin{array}{c}35 \\
(38.89)\end{array}$ & $\begin{array}{c}11 \\
(12.22)\end{array}$ & 2.48 & IV & $\begin{array}{c}11 \\
(40.74)\end{array}$ & $\begin{array}{c}15 \\
(55.56)\end{array}$ & $\begin{array}{c}1 \\
(3.70)\end{array}$ & 2.37 & VII \\
\hline 13 & For chatting & $\begin{array}{c}35 \\
(38.89\end{array}$ & $\begin{array}{c}32 \\
(35.55)\end{array}$ & $\begin{array}{c}23 \\
(25.55)\end{array}$ & 2.13 & XVI & $\begin{array}{c}10 \\
(37.04)\end{array}$ & $\begin{array}{c}12 \\
(44.44)\end{array}$ & $\begin{array}{c}5 \\
(18.52)\end{array}$ & 2.18 & $X V$ \\
\hline
\end{tabular}


Contd...

\begin{tabular}{|c|c|c|c|c|c|c|c|c|c|c|c|}
\hline 14 & Just for pass time & $\begin{array}{c}13 \\
(14.44)\end{array}$ & $\begin{array}{c}31 \\
(34.44)\end{array}$ & $\begin{array}{c}46 \\
(51.11)\end{array}$ & 1.63 & XXVII & $\begin{array}{c}3 \\
(11.11)\end{array}$ & $\begin{array}{c}15 \\
(55.56)\end{array}$ & $\begin{array}{c}9 \\
(33.33)\end{array}$ & 1.78 & XXI \\
\hline 15 & $\begin{array}{l}\text { For matrimonial } \\
\text { purpose }\end{array}$ & $\begin{array}{c}16 \\
(17.78)\end{array}$ & $\begin{array}{c}44 \\
(48.89)\end{array}$ & $\begin{array}{c}30 \\
(33.33)\end{array}$ & 1.84 & XXIV & $\begin{array}{c}3 \\
(11.11)\end{array}$ & $\begin{array}{c}8 \\
(29.63)\end{array}$ & $\begin{array}{c}16 \\
(59.26)\end{array}$ & 1.52 & XXVII \\
\hline 16 & $\begin{array}{l}\text { For sending } \\
\text { messages }\end{array}$ & $\begin{array}{c}26 \\
(28.89)\end{array}$ & $\begin{array}{c}41 \\
(45.55)\end{array}$ & $\begin{array}{c}23 \\
(25.56)\end{array}$ & 2.03 & $\mathrm{XX}$ & $\begin{array}{c}7 \\
(25.93)\end{array}$ & $\begin{array}{c}17 \\
(62.96)\end{array}$ & $\begin{array}{c}3 \\
(11.11)\end{array}$ & 2.15 & XVI \\
\hline 17 & $\begin{array}{l}\text { For telephony } \\
\text { communication }\end{array}$ & $\begin{array}{c}24 \\
(26.67)\end{array}$ & $\begin{array}{c}22 \\
(24.44)\end{array}$ & $\begin{array}{c}44 \\
(48.89)\end{array}$ & 1.78 & XXV & $\begin{array}{c}5 \\
(18.52)\end{array}$ & $\begin{array}{c}7 \\
(25.92)\end{array}$ & $\begin{array}{c}15 \\
(55.56)\end{array}$ & 1.63 & XXV \\
\hline 18 & $\begin{array}{l}\text { To develop own } \\
\text { website }\end{array}$ & $\begin{array}{c}18 \\
(20.0)\end{array}$ & $\begin{array}{c}41 \\
(45.56)\end{array}$ & $\begin{array}{c}31 \\
(34.44)\end{array}$ & 1.85 & XXIII & $\begin{array}{c}5 \\
(18.52)\end{array}$ & $\begin{array}{c}8 \\
(29.63)\end{array}$ & $\begin{array}{c}14 \\
(51.85)\end{array}$ & 1.67 & XXIV \\
\hline 19 & $\begin{array}{l}\text { To collect } \\
\text { information for } \\
\text { class notes }\end{array}$ & $\begin{array}{c}41 \\
(45.56)\end{array}$ & $\begin{array}{c}32 \\
(35.56)\end{array}$ & $\begin{array}{c}17 \\
(18.89)\end{array}$ & 2.27 & XI & $\begin{array}{c}4 \\
(14.81)\end{array}$ & $\begin{array}{c}9 \\
(33.33)\end{array}$ & $\begin{array}{c}14 \\
(51.85)\end{array}$ & 1.63 & XXVI \\
\hline 20 & To satisfy curiosity & $\begin{array}{c}27 \\
(30.00)\end{array}$ & $\begin{array}{c}42 \\
(46.67)\end{array}$ & $\begin{array}{c}21 \\
(23.33)\end{array}$ & 2.07 & XVII & $\begin{array}{c}11 \\
(40.74)\end{array}$ & $\begin{array}{c}15 \\
(55.56)\end{array}$ & $\begin{array}{c}1 \\
(3.70)\end{array}$ & 2.37 & VIII \\
\hline 21 & For education & $\begin{array}{c}54 \\
(60.00)\end{array}$ & $\begin{array}{c}28 \\
(31.11)\end{array}$ & $\begin{array}{c}8 \\
(8.89)\end{array}$ & 2.51 & III & $\begin{array}{c}10 \\
(37.03)\end{array}$ & $\begin{array}{c}16 \\
(59.26)\end{array}$ & $\begin{array}{c}1 \\
(3.70)\end{array}$ & 2.33 & $\mathrm{X}$ \\
\hline 22 & $\begin{array}{l}\text { To communicate } \\
\text { with others }\end{array}$ & $\begin{array}{c}34 \\
(37.78)\end{array}$ & $\begin{array}{c}42 \\
(46.67) \\
\end{array}$ & $\begin{array}{c}14 \\
(15.55)\end{array}$ & 2.22 & $X V$ & $\begin{array}{c}23 \\
(85.19) \\
\end{array}$ & $\begin{array}{c}4 \\
(14.81)\end{array}$ & $\begin{array}{c}0 \\
(0.00)\end{array}$ & 2.85 & I \\
\hline 23 & $\begin{array}{l}\text { To prepare for } \\
\text { examination }\end{array}$ & $\begin{array}{c}36 \\
(40.00)\end{array}$ & $\begin{array}{c}42 \\
(46.67)\end{array}$ & $\begin{array}{c}12 \\
(13.33)\end{array}$ & 2.27 & XI & $\begin{array}{c}18 \\
(66.67)\end{array}$ & $\begin{array}{c}8 \\
(29.63)\end{array}$ & $\begin{array}{c}1 \\
(3.70)\end{array}$ & 2.63 & III \\
\hline 24 & $\begin{array}{l}\text { To update } \\
\text { knowledge }\end{array}$ & $\begin{array}{c}53 \\
(58.89) \\
\end{array}$ & $\begin{array}{c}25 \\
(27.78) \\
\end{array}$ & $\begin{array}{c}12 \\
(13.33) \\
\end{array}$ & 2.45 & $\mathrm{~V}$ & $\begin{array}{c}6 \\
(22.22) \\
\end{array}$ & $\begin{array}{c}20 \\
(74.07)\end{array}$ & $\begin{array}{c}1 \\
(3.70)\end{array}$ & 2.18 & $\mathrm{XV}$ \\
\hline 25 & To read news & $\begin{array}{c}39 \\
(43.33)\end{array}$ & $\begin{array}{c}36 \\
(40.00)\end{array}$ & $\begin{array}{c}15 \\
(16.67)\end{array}$ & 2.26 & XIII & $\begin{array}{c}11 \\
(40.74)\end{array}$ & $\begin{array}{c}15 \\
(55.55)\end{array}$ & $\begin{array}{c}1 \\
(3.70)\end{array}$ & 2.37 & IX \\
\hline 26 & $\begin{array}{l}\text { To download } \\
\text { software }\end{array}$ & $\begin{array}{c}32 \\
(35.56)\end{array}$ & $\begin{array}{c}28 \\
(31.11)\end{array}$ & $\begin{array}{c}30 \\
(33.33)\end{array}$ & 2.02 & XXII & $\begin{array}{c}8 \\
(29.63)\end{array}$ & $\begin{array}{c}15 \\
(55.55)\end{array}$ & $\begin{array}{c}4 \\
(14.81)\end{array}$ & 2.15 & XVII \\
\hline 27 & To purchase items & $\begin{array}{c}16 \\
(17.78)\end{array}$ & $\begin{array}{c}28 \\
(31.11)\end{array}$ & $\begin{array}{c}46 \\
(51.11)\end{array}$ & 1.67 & XXVI & $\begin{array}{c}8 \\
(29.63)\end{array}$ & $\begin{array}{c}4 \\
(14.81)\end{array}$ & $\begin{array}{c}15 \\
(55.56)\end{array}$ & 1.74 & XXII \\
\hline
\end{tabular}

$\mathrm{r}_{\mathrm{s}}=0.63 \quad \mathrm{t}=4.01$

Rank correlation coefficient $\left(r_{s}\right)=0.37 \quad t=4.01$ significant at 0.01 level of probability)

Tabulated value of $t$ at 0.01 level of probability with 25 degrees of freedom $=2.79$

The findings revealed that there is a significant correlation between the male and female agricultural research scholars with regard to their purpose of using internet, this might be due to the reason that both male and female agricultural research scholars are in the same situation, same generation having same ambition and target as students and using the internet facilities for class notes, communicate to each, for research etc.

These findings are in line with the findings of Kumar and Kaur (2006). The findings are not in line with the findings of Garhwal (2010), Mishra et al., (2011) and Anunobi (2006). 


\section{Preference of location for access to internet}

The data in table 2 depicted that the male agricultural research scholars mostly preferred college library (MS 2.50) for internet access which was ranked first followed by hostel (MS 1.97), Department (MS 1.91), private cyber cafe (MS 1.73), home (MS 1.57), friends (MS 1.24) and neighborhood (1.14) which were ranked second, third, fourth, fifth, sixth and seventh respectively. Whereas, the female agricultural research scholars mostly preferred college department (MS 2.04) for internet access which was ranked first followed by college library (MS 1.96), Home (MS 1.78), friends (MS 1.52) and hostel (MS1.18) which were ranked second, third, fourth, fifth and sixth respectivel. No female agricultural research scholars were used cyber café for internet access. It can be observed from the findings that the best source/ place accessed by the respondents were college library and department, which might be due to the reason that since the sufficient internet facility was available at college library, hostel and department, the students preferred these places for internet access. These findings are in line with the findings of Mulimani and Gudimani (2008) and Garhwal (2010) whereas the findings are not in line with the findings of Anunobi (2006). The value of rank order correlation $\left(\mathrm{r}_{\mathrm{s}}\right)$ was found to be 0.25 for which the calculated values of ' $t$ ' $(0.58)$ was less than its tabulated value at 5 per cent level of significance. Hence the null hypothesis $\left(\mathrm{H}_{02.5}\right)$ was accepted and alternate hypothesis was rejected. This leads to the conclusion that there is no significant correlation between the male and female agricultural research scholars with regard to their preference of location for access to Internet.
In conclusion, majority of male agricultural research scholars preferred college library for internet access, used internet to collect information for class assignments and preferred the internet for getting information as compared to other media. Whereas female agricultural research scholars preferred mostly department for internet access, had used internet without any expenditure, used internet mostly to communicate with others, and preferred the newspaper for getting information as compared to other media.

\section{References}

Anunobi, C.V. 2006. Dynamics of internet usage: A case of students of the federal university of technology Owerri (FUTO) Nigeria. Educational research and reviews, 1:192-195

Garhwal, S. 2010. Internet utilization behavior of Agricultural students of Swami Keshwanand Rajasthan agricultural University, Bikaner M.sc. (Ag) Thesis.

Kumar, R. and Kaur, A. 2006. Internet use by teachers and students in Engineering colleges of Punjab, Haryana and Himachal Pradesh States of India: An analysis" Electronic Journal of Academic and Special Librarianship vol.7:61-63

Mishra, O.P, Kumar, S, Dash, D.K and Kumar, R. 2011. Under Graduate Agriculture Students and Uses of the Internet: Case of Banaras Hindu University". JCS, vol 29 April 2011

Rehman, K. U, Hunjra, A, I. Safwan, N and Ahmed, A. 2010. Students' attitude towards the uses of internet, International Journal of Business and Management, vol. 5, No. 6.June 2010.

\section{How to cite this article:}

Choudhary, L.R., B.S. Bhimawat and Chamandeep Kaur. 2019. Purpose of using Internet and Preference of Location for Access to Internet by Agricultural Research Scholars while using Internet of Agriculture Science in MPUAT, Udaipur (Rajasthan). Int.J.Curr.Microbiol.App.Sci. 8(02): 1519-1524. doi: https://doi.org/10.20546/ijcmas.2019.802.176 